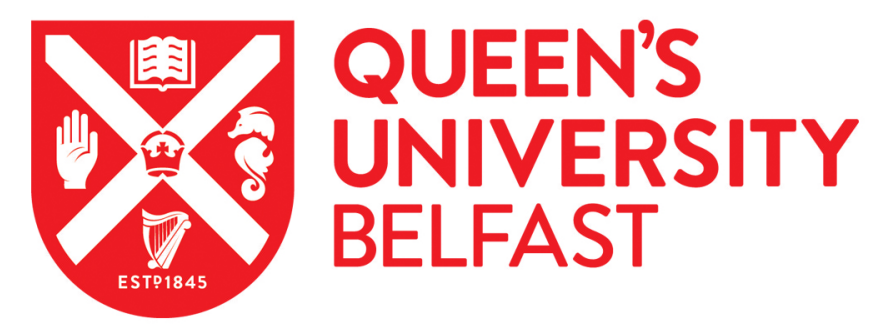

\title{
Scaling of magneto-quantum-radiative hydrodynamic equations: From laser-produced plasmas to astrophysics
}

Cross, J. E., Reville, B., \& Gregori, G. (2014). Scaling of magneto-quantum-radiative hydrodynamic equations: From laser-produced plasmas to astrophysics. Astrophysical Journal, 795, [59]. https://doi.org/10.1088/0004$637 X / 795 / 1 / 59$

Published in:

Astrophysical Journal

Document Version:

Publisher's PDF, also known as Version of record

Queen's University Belfast - Research Portal:

Link to publication record in Queen's University Belfast Research Portal

Publisher rights

Copyright 2014. The American Astronomical Society. All rights reserved. Printed in the U.S.A.

\section{General rights}

Copyright for the publications made accessible via the Queen's University Belfast Research Portal is retained by the author(s) and / or other copyright owners and it is a condition of accessing these publications that users recognise and abide by the legal requirements associated with these rights.

Take down policy

The Research Portal is Queen's institutional repository that provides access to Queen's research output. Every effort has been made to ensure that content in the Research Portal does not infringe any person's rights, or applicable UK laws. If you discover content in the Research Portal that you believe breaches copyright or violates any law, please contact openaccess@qub.ac.uk. 


\title{
SCALING OF MAGNETO-QUANTUM-RADIATIVE HYDRODYNAMIC EQUATIONS: FROM LASER-PRODUCED PLASMAS TO ASTROPHYSICS
}

\author{
J. E. Cross ${ }^{1}$, B. Reville ${ }^{2}$, AND G. GRegOrI ${ }^{1}$ \\ ${ }^{1}$ Clarendon Laboratory, University of Oxford, Parks Road, Oxford OX1 3PU, UK; j.e.cross@physics.ox.ac.uk \\ ${ }^{2}$ Centre for Plasma Physics, Queen's University Belfast, University Road, Belfast BT7 1NN, UK \\ Received 2014 January 30; accepted 2014 September 5; published 2014 October 14
}

\begin{abstract}
We introduce the equations of magneto-quantum-radiative hydrodynamics. By rewriting them in a dimensionless form, we obtain a set of parameters that describe scale-dependent ratios of characteristic hydrodynamic quantities. We discuss how these dimensionless parameters relate to the scaling between astrophysical observations and laboratory experiments.
\end{abstract}

Key words: hydrodynamics - ISM: jets and outflows - magnetohydrodynamics (MHD) - plasmas radiative transfer - supernovae: general

\section{INTRODUCTION}

The study of astrophysical phenomena using laser-produced plasma is a growing field of research (Remington et al. 1999; Gregori et al. 2012; Savin et al. 2012; Meinecke et al. 2014). Modern laser facilities can deliver large amounts of energy in very short times, exceeding what is possible from more conventional techniques such as gas guns or pulsed power machines. Pressure near the laser spot (where most of the laser energy is deposited) can reach values in excess of tens of megabars and is comparable to the energy density of bound electrons in atoms. Under these conditions, quantum processes and radiation diffusion can also become important. Return currents as well as steep density and pressure gradients produce magnetic fields (Haines 1986a) that can modify the overall transport of charged particles. These large deposited energies then drive powerful shock waves into the ambient medium (Foster et al. 2002; Robey et al. 2002; Klein et al. 2003; Hansen et al. 2005). The process bears similarities to many astrophysical phenomena where energy is impulsively released into the interstellar medium, such as supernova remnants (Chevalier et al. 1992), Herbig-Haro flows (Hartigan et al. 1987), and accretion shocks (Miniati et al. 2000).

Laboratory experiments offer a viable complementary approach to both astrophysical observations (by providing, for example, the means of directly measuring quantities of interest not accessible by observation) and numerical calculations, thus overcoming limitations in resolution and numerical viscosity, and potentially addressing nonlinear aspects of the dynamical evolution or validating simulation codes.

This is meaningful only if the relevant physics in the laboratory is related to the astrophysical object. We refer to this as a similarity relation between the two systems. The most obvious situation is one where the laboratory experiment reaches the exact conditions found in the astrophysical object. This has been exploited, for example, to study the equation of state of planetary interiors (Jeanloz et al. 2007) and other compact objects (Kritcher et al. 2008; García Saiz et al. 2008). However, it is not always possible to reach the exact conditions that we are interested in because the spatial, temporal, and energy scales may be outside the range of what is directly reproducible in an experiment. A similarity relation still exists if we can show that the laboratory and astrophysical systems evolve in a way that the governing equations are invariant under a scale transformation; this requires the corresponding spatial, density, pressure, time, and so on, values in one system to be mapped onto the other system by multiplicative constants. This similarity can be obtained via fluid equations (Ryutov et al. 1999) or even at the kinetic level (Connor \& Taylor 1977; Ryutov et al. 2012) under some conditions. This paper concerns the magnetohydrodynamic (MHD) similarity and provides a general framework to include effects arising from finite resistivity, thermal conduction, radiation diffusion, and quantum nonlocality.

Fluid similarity has previously been discussed, quite extensively, by Ryutov and Falize (Ryutov et al. 1999, 2000, 2001, 2012; Falize et al. 2011a, 2011b). However, only selected aspects of the full governing equations have been investigated in the previous work, i.e., viscous hydrodynamics, radiative effects, or resistive MHD. The aim of the present work is thus to bring all of the different elements of the equations together in a simple conceptual form. We consider here the most general form of the fluid equations, including magnetic, radiative, and quantum effects, which are therefore applicable to a wide range of cases. By rewriting these equations in a dimensionless form we derive a set of characteristic ratios containing the details of the microscopic properties of the fluid at a given scale. Values of these ratios tell us how important local properties are in determining the overall fluid motion.

We introduce the full set of fluid equations in Section 2 and specialize them for the case of an optically thick plasma in Section 3. In Section 3 we also discuss the Bohm potential and the inclusion of quantum dynamics in the fluid model. This can become important for exotic matter, such as inside neutron stars or white dwarfs. Section 4 describes the dimensionless analysis, and in Section 5 we derive the dimensionless fluid equations for the optically thick case. The optically thin equations are instead given in Section 6. Section 7 discusses the different dimensionless numbers and their relevance to experiments. In Section 8 we compare some laboratory experiments with their astrophysical counterparts and apply similarity in the context of our dimensionless analysis. We draw our conclusions in Section 9.

\section{GENERAL EQUATIONS}

Although the equations of fluid dynamics are the same everywhere in the universe, there is no guarantee that a laboratory fluid would behave in the same manner as an astrophysical fluid. 
The two systems will exhibit the same dynamics only under some specific conditions. In order to explicitly extract such relations, we first write the full set of MHD equations in the presence of heat conduction, radiation diffusion, and quantum effects (Zeldovich et al. 1966; Drake 2006; McClarren et al. 2010; Haas 2011). We assume the plasma is described by a single fluid but with appropriate transport coefficients that are derived from kinetic theory (Chapman \& Cowling 1970).

\subsection{Continuity Equation}

The equation for the conservation of mass is given by:

$$
\frac{\partial \rho}{\partial t}+\nabla \cdot \rho \boldsymbol{u}=0
$$

where $\rho$ is the mass density, $t$ the time, and $\boldsymbol{u}$ the fluid velocity.

\subsection{Momentum Equation}

The equation for conservation of momentum reads as

$$
\rho\left(\frac{\partial \boldsymbol{u}}{\partial t}+\boldsymbol{u} \cdot \nabla \boldsymbol{u}\right)=-\nabla p+\Phi_{\mathrm{Bohm}}+\nabla \cdot \boldsymbol{\sigma}_{v}+\boldsymbol{F}_{\mathrm{EM}}+f_{\mathrm{rad}}
$$

where $p$ is the fluid (ram) pressure, $\Phi_{\text {Bohm }}$ the quantum Bohm potential, $\boldsymbol{\sigma}_{v}$ the stress tensor, $\boldsymbol{F}_{\mathrm{EM}}$ electromagnetic volume forces (the interaction of charged particles and electromagnetic (EM) fields), and $f_{\text {rad }}$ the volume force of radiation on matter. This equation shows that, in the most general case, the momentum associated with a fluid element can change not only by the inertial term and the pressure gradient but also because of exchange effects (the Bohm potential contribution), viscous drag, and radiative forces. Each one of these nonideal terms will be discussed in detail in the following sections. We also note that in Equation (1b) the radiation force on matter, $f_{\text {rad }}$, in its most general form, includes effects from absorption and scattering (Shu 1992).

\subsection{Energy Equation}

The equation for conservation of energy is

$$
\begin{aligned}
& \frac{\partial}{\partial t}\left(\rho \epsilon+\frac{\rho u^{2}}{2}+E_{R}\right)+\nabla \cdot\left[\rho \boldsymbol{u}\left(\epsilon+\frac{u^{2}}{2}\right)+p \boldsymbol{u}\right] \\
& \quad=-\nabla \cdot \boldsymbol{H}-\boldsymbol{J} \cdot \boldsymbol{E}+\Phi_{\mathrm{Bohm}} \cdot \boldsymbol{u}-f_{\mathrm{rad}} \cdot \boldsymbol{u},
\end{aligned}
$$

where $\epsilon$ is the specific internal energy, $E_{R}$ the energy density of the radiation field, $\boldsymbol{H}$ the energy flux from nonideal terms, $\boldsymbol{J}$ the current density, and $\boldsymbol{E}$ the electric field. The nonideal energy flux is

$$
\boldsymbol{H}=\boldsymbol{F}_{R}+\left(p_{R}+E_{R}\right) \boldsymbol{u}+\boldsymbol{Q}-\boldsymbol{\sigma}_{v} \cdot \boldsymbol{u},
$$

where $\boldsymbol{F}_{R}$ is the radiative energy flux, $p_{R}$ the radiation pressure, and $\boldsymbol{Q}$ the heat flux. Here we have distinguished between the radiative enthalpy flux associated with the matter motion, $\left(E_{R}+p_{R}\right) \boldsymbol{u}$, and the radiative energy flux in the rest frame of the fluid, $F_{R}$ (see discussion in Shu 1992).

Differently from previous work, the above equations correctly describe quantum effects, which become important for highdensity fluids (Schmidt et al. 2012), when the number density reaches values $\gtrsim 10^{24} \mathrm{~cm}^{-3}$, as in white dwarfs or neutron star matter or at small scales. This means that Pauli blocking, tunneling, and wave-packet spreading begin to exert an effective quantum pressure to the system (Haas 2011). This approach follows from the fact that deterministic equations can be used
Table 1

Numerical Values for the Braginskii Coefficients for Various Values of $Z$, Adapted from Braginskii (1965)

\begin{tabular}{llllll}
\hline \hline & $Z=1$ & $Z=2$ & $Z=3$ & $Z=4$ & $Z \rightarrow \infty$ \\
\hline$\beta_{0}^{\prime \prime}$ & 3.053 & 1.784 & 1.442 & 1.285 & 0.877 \\
$\delta_{0}$ & 3.7703 & 1.0465 & 0.5814 & 0.4106 & 0.0961 \\
$\beta_{1}^{\prime \prime}$ & 1.5 & 1.5 & 1.5 & 1.5 & 1.5 \\
$\delta_{1}$ & 14.79 & 10.80 & 9.618 & 9.055 & 7.482 \\
\hline
\end{tabular}

to describe both single-particle and many-body distribution functions in the quantum limit if an appropriate potential is introduced in the hydrodynamic equations (Bohm 1952; Mostacci et al. 2008). See Section 3.1 below for more detail.

\subsection{Induction Equation}

Starting from Ohm's law, and neglecting displacement current, we obtain

$$
\begin{aligned}
\frac{\partial \boldsymbol{B}}{\partial t}= & \nabla \times(\boldsymbol{u} \times \boldsymbol{B})+\eta \nabla^{2} \boldsymbol{B}+\frac{m}{e(1+Z)} \frac{\nabla p \times \nabla \rho}{\rho^{2}} \\
& +\nabla \times\left(\boldsymbol{B} \times \frac{\tau_{e i}}{m_{e}} \frac{\beta_{1}^{\prime \prime} \chi^{2}+\beta_{0}^{\prime \prime}}{\Delta} \nabla T\right),
\end{aligned}
$$

where $\boldsymbol{B}$ is the magnetic field, $\eta$ the magnetic diffusivity ( $\eta=1 / \sigma_{0} \mu_{0}$ where $\sigma_{0}$ is the electric conductivity and $\mu_{0}$ the vacuum permeability), $m$ the average mass per particle, $m_{e}$ the electron mass, $e$ the elementary charge, $Z$ the degree of ionization, $\tau_{e i}$ the electron-ion collision time, $\Delta=\chi^{4}+\delta_{1} \chi^{2}+\delta_{0}$ (where $\chi=\omega_{c e} \tau_{e i}$ is the Hall parameter and $\omega_{c e}$ is the electron cyclotron frequency), $T$ the fluid temperature, and $\beta_{0}^{\prime \prime}, \beta_{1}^{\prime \prime}, \delta_{0}$, and $\delta_{1}$ are Braginskii coefficients (Braginskii 1965). Values for the Braginskii coefficients are given in Table 1.

In addition to magnetic diffusion (second term on the righthand side of Equation (1e)), we have written the induction equation to include baroclinic generation of the magnetic field via the Biermann battery mechanism (Biermann 1950; Kulsrud $\&$ Zweibel 2008) and advection of the magnetic field due to the Nernst effect (Haines 1986a). These are the last two terms on the right-hand side of Equation (1e), respectively. In many laboratory and astrophysical scenarios, these terms represent the next-highest-order correction to Ohm's law (Haines 1986b; Nishiguchi et al. 1985). Although Ohm's law contains several additional terms (Haines 1986a), here we restrict it to the case of small magnetic fields, where zeroth-order (Biermann battery) and first-order (Nernst) terms have been shown to be the dominant mechanism for magnetic field generation in many plasma experiments (Manuel et al. 2013).

\section{OPTICALLY THICK SOURCE TERMS}

Under the conditions of optically thick radiation, the source terms in Equations (1b), (1c), and (1d) are explicitly given by

$$
\begin{gathered}
-\nabla p_{R}=f_{\mathrm{rad}} \\
p_{R}=\frac{E_{R}}{3}=\frac{4 \sigma T^{4}}{3 c} \\
\Phi_{\mathrm{Bohm}}=\frac{\hbar^{2} \rho}{2 m_{e} m_{i}} \nabla\left(\frac{\nabla^{2} \sqrt{\rho}}{\sqrt{\rho}}\right)
\end{gathered}
$$




$$
\begin{gathered}
\boldsymbol{\sigma}_{v}=\rho v\left[\nabla \boldsymbol{u}+(\nabla \boldsymbol{u})^{T}-\frac{2}{3}(\nabla \cdot \boldsymbol{u}) \underline{\boldsymbol{I}}\right]+\zeta(\nabla \cdot \boldsymbol{u}) \underline{\boldsymbol{I}} \\
\boldsymbol{F}_{\mathrm{EM}}=\rho_{C} \boldsymbol{E}+\boldsymbol{J} \times \boldsymbol{B} \\
\boldsymbol{F}_{R}=-\frac{16 \sigma T^{3}}{3 \kappa_{R} \rho} \nabla T \\
\boldsymbol{Q}=-\kappa_{\mathrm{th}} \nabla T=-\chi_{\mathrm{th}} \rho c_{p} \nabla T \\
=-\frac{\chi_{\mathrm{th}} \rho k_{B} \gamma}{m(\gamma-1)} \nabla T
\end{gathered}
$$

where $\sigma$ is the Stefan-Boltzmann constant, $c$ the speed of light, $\hbar$ the reduced Planck's constant, $m_{i}$ the ion mass, $v$ the kinematic viscosity with $v=\mu / \rho$, with $\mu$ being the (dynamic) viscosity, $\underline{I}$ the identity tensor, and $\zeta$ the second coefficient of viscosity, $\rho_{C}$ the charge density, $\kappa_{R}$ the Rosseland mean opacity, $\kappa_{\text {th }}$ the coefficient of heat conduction, $\chi_{\text {th }}$ the kinematic coefficient of thermal diffusivity, $c_{p}$ the specific heat capacity at constant pressure, $k_{B}$ Boltzmann's constant, and $\gamma$ the adiabatic index.

When the fluid is optically thick, we can reduce the pressure tensor to a scalar radiation pressure and write it in terms of an isotropic energy density. Equation (2a) is thus only applicable in this limit. Equation (2b) represents the isotropic thermal radiation pressure within the plasma and the related energy density of that radiation assuming a Planck distribution (Castor 2004). Equation (2f) gives the radiative energy flux, within the local thermodynamic equilibrium (LTE) approximation. In this form, it corresponds to the Rosseland heat flux (Castor 2004; Drake 2006). Clearly, these equations are not applicable to the case of optically thin systems or when there is an optically thin preshock material but optically thick postshock material (McClarren et al. 2010). We will discuss the optically thin case in Section 6 below.

The quantum potential is explicitly given in Equation (2c). A derivation of this term is given in Section 3.1. Equation (2d) gives the form of the stress tensor. This form does not assume that the fluid is incompressible, i.e., $\nabla \cdot \boldsymbol{u}$ does not have to be equal to zero (Drake 2006). It also considers the effects of viscosity to the second order. Equation (2e) defines the electromagnetic (Lorentz) force on the system in standard form. Finally, Equation (2g) describes the thermal heat flux in the diffusive limit (Landau \& Lifshitz 1959).

\subsection{Quantum Potential}

Given the presence of the Bohm potential in the above equations and the fact that this term is often omitted, it is important to give a detailed explanation and derivation of its appearance. It arises from rewriting the Schrödinger equation in polar form with a wavefunction given by

$$
\phi=R e^{i S / \hbar}
$$

where $R$ and $S$ are real-valued functions. The Schrödinger equation can be thus divided into an imaginary part

$$
\frac{\partial R}{\partial t}=-\frac{1}{2 m}\left(R \nabla^{2} S+2 \nabla R \cdot \nabla S\right)
$$

and a real part

$$
\frac{\partial S}{\partial t}=-\left[\frac{(\nabla S)^{2}}{2 m}+V+Q\right]
$$

where $V$ is the external potential and

$$
Q=-\frac{\hbar^{2}}{2 m} \frac{\nabla^{2} R}{R}
$$

If we now identify, using the correspondence to the classical limit, $R^{2}=\rho$, and $\mathbf{u}=\nabla S / m$, then Equation (3) can be reexpressed as a continuity equation, and Equation (4) has the form of an energy equation with the classical potential corrected by the quantum term $Q$. This leads, for example, to the inclusion of $\rho Q / m$ as an energy-density correction in the momentum equation.

In general the equations with quantum potential correction are written separately for the ion and electron species (Haas 2011). For simplicity, we start by considering the case of an ideal fluid where the source terms are only the pressure gradient and electromagnetic forces:

$$
\begin{aligned}
\frac{\partial \boldsymbol{u}_{e}}{\partial t}+\boldsymbol{u}_{e} \cdot \nabla \boldsymbol{u}_{e}= & -\frac{\nabla p_{e}}{m_{e} n_{e}}-\frac{e}{m_{e}}\left(\boldsymbol{E}+\boldsymbol{u}_{e} \times \boldsymbol{B}\right) \\
& +\frac{\hbar^{2}}{2 m_{e}^{2}} \nabla\left(\frac{\nabla^{2} \sqrt{n_{e}}}{\sqrt{n_{e}}}\right), \\
\frac{\partial \boldsymbol{u}_{i}}{\partial t}+\boldsymbol{u}_{i} \cdot \nabla \boldsymbol{u}_{i}= & -\frac{\nabla p_{i}}{m_{i} n_{i}}+\frac{e}{m_{i}}\left(\boldsymbol{E}+\boldsymbol{u}_{i} \times \boldsymbol{B}\right) \\
& +\frac{\hbar^{2}}{2 m_{i}^{2}} \nabla\left(\frac{\nabla^{2} \sqrt{n_{i}}}{\sqrt{n_{i}}}\right),
\end{aligned}
$$

where $n_{e}\left(n_{i}\right), \boldsymbol{u}_{\boldsymbol{e}}\left(\boldsymbol{u}_{\boldsymbol{i}}\right)$, and $p_{e}\left(p_{i}\right)$ are the electron (ion) number density, velocity, and pressure, respectively. Quantities with no subscript are instead used to describe average fluid properties. By defining an average mass density and fluid velocity as

$$
\rho=m_{e} n_{e}+m_{i} n_{i}, \quad \boldsymbol{u}=\frac{m_{e} n_{e} \boldsymbol{u}_{e}+m_{i} n_{i} \boldsymbol{u}_{i}}{m_{e} n_{e}+m_{i} n_{i}},
$$

we can combine Equations (5a) and (5b) into a single fluid description by multiplying each one by $n_{e} m_{e}$ and $n_{i} m_{i}$, respectively, and by adding them together. The resulting momentum equation is

$$
\rho\left(\frac{\partial \boldsymbol{u}}{\partial t}+\boldsymbol{u} \cdot \nabla \boldsymbol{u}\right)=-\nabla p+\boldsymbol{J} \times \boldsymbol{B}+\frac{\hbar^{2} \rho}{2 m_{e} m_{i}} \nabla\left(\frac{\nabla^{2} \sqrt{\rho}}{\sqrt{\rho}}\right)
$$

which has the same form of the quantum potential as seen in Equation (2c). In this derivation we have assumed quasineutrality and taken the total pressure to be the sum of the electron and ion pressures, that is, $p_{e}+p_{i}=p$.

\section{DIMENSIONLESS ANALYSIS}

We now rescale the variables in the hydrodynamic equations by a corresponding characteristic value. This allows us to rewrite the equations in an invariant form, and the detail associated with the physical dimensions of the system is contained in a series of dimensionless numbers, which represent ratios of those characteristic values. We write the velocity, position, time, and density as

$$
\boldsymbol{u} \rightarrow u_{0} \boldsymbol{u}^{*}, \quad \boldsymbol{r} \rightarrow \ell_{0} \boldsymbol{r}^{*}, \quad t \rightarrow \frac{\ell_{0}}{u_{0}} t^{*}, \quad \rho \rightarrow \rho_{0} \rho^{*},
$$


where $u_{0}, \ell_{0}$, and $\rho_{0}$ are the characteristic velocity, length, and density of the system, respectively. From now on we will use the convention that starred quantities (i.e., $\boldsymbol{u}^{*}$ ) are dimensionless, and quantities with subscript $O$ (i.e., $u_{0}$ ) correspond to a characteristic value for that variable. The above assumptions imply

$$
\frac{\partial}{\partial t} \rightarrow \frac{u_{0}}{\ell_{0}} \frac{\partial}{\partial t^{*}}, \quad \nabla \rightarrow \frac{\nabla^{*}}{\ell_{0}} .
$$

Similarly, we can set

$$
p \rightarrow p_{0} p^{*}, \quad \boldsymbol{B} \rightarrow B_{0} \boldsymbol{B}^{*}, \quad \epsilon \rightarrow \epsilon_{0} \epsilon^{*} .
$$

However, the choice of the values for $p_{0}, B_{0}$, and $\epsilon_{0}$ is not arbitrary. To see this, we consider the momentum Equation (1b) but with the only source terms being the pressure gradient and magnetic field (i.e., in the ideal MHD limit). Using the relation $\boldsymbol{J} \times \boldsymbol{B}=(\boldsymbol{B} \cdot \nabla) \boldsymbol{B} / \mu_{0}-\nabla\left(B^{2} / 2 \mu_{0}\right)$, we can rewrite the momentum Equation (1b) as

$$
\begin{gathered}
\rho_{0} \rho^{*}\left(\frac{u_{0}}{\ell_{0}} \frac{\partial u_{0} \boldsymbol{u}^{*}}{\partial t^{*}}+u_{0} \boldsymbol{u}^{*} \cdot \frac{\nabla^{*}}{\ell_{0}} u_{0} \boldsymbol{u}^{*}\right)=-\frac{\nabla^{*}}{\ell_{0}} p_{0} p^{*} \\
+\frac{B_{0}^{2}}{\ell_{0} \mu_{0}}\left[\left(\boldsymbol{B}^{*} \cdot \nabla^{*}\right) \boldsymbol{B}^{*}-\nabla^{*} \frac{B^{* 2}}{2}\right] .
\end{gathered}
$$

Noticing the common factor of $u_{0}^{2} \rho_{0} / \ell_{0}$ on the left and dividing through gives

$$
\begin{gathered}
\rho^{*}\left(\frac{\partial \boldsymbol{u}^{*}}{\partial t^{*}}+\boldsymbol{u}^{*} \cdot \nabla^{*} \boldsymbol{u}^{*}\right)=-\frac{p_{0}}{\rho_{0} u_{0}^{2}} \nabla^{*} p^{*} \\
+\frac{B_{0}^{2}}{\mu_{0} \rho_{0} u_{0}^{2}}\left[\left(\boldsymbol{B}^{*} \cdot \nabla^{*}\right) \boldsymbol{B}^{*}-\nabla^{*} \frac{B^{* 2}}{2}\right] .
\end{gathered}
$$

Because we require this equation to have the same form as Equation (1b), that is, to be invariant under the scaling transformation, this means that $p_{0} \equiv \rho_{0} u_{0}^{2}$ and $B_{0} \equiv u_{0} \sqrt{\mu_{0} \rho_{0}}$. We see that the reference magnetic field has a value such that the fluid velocity and the Alfvén velocity (Alfvén 1942) are the same.

We can follow a similar procedure to determine the value for $\epsilon_{0}$. Using the energy Equation (1c) in the ideal case with no source terms, we get

$$
\begin{aligned}
& \frac{u_{0}}{\ell_{0}} \frac{\partial}{\partial t^{*}}\left(\rho_{0} \epsilon_{0} \rho^{*} \epsilon^{*}+\frac{\rho_{0} u_{0}^{2} \rho^{*} u^{* 2}}{2}\right) \\
& \quad=-\frac{\nabla^{*}}{\ell_{0}} \cdot\left[\rho_{0} u_{0} \rho^{*} \boldsymbol{u}^{*}\left(\epsilon_{0} \epsilon^{*}+\frac{u_{0}^{2} u^{* 2}}{2}\right)+\rho_{0} u_{0}^{2} p^{*} u_{0} \boldsymbol{u}^{*}\right]
\end{aligned}
$$

Dividing through by a factor of $u_{0}^{3} \rho_{0} / \ell_{0}$ we obtain

$$
\begin{aligned}
& \frac{\partial}{\partial t^{*}}\left(\frac{\epsilon_{0}}{u_{0}^{2}} \rho^{*} \epsilon^{*}+\frac{\rho^{*} u^{* 2}}{2}\right) \\
& \quad=-\nabla^{*} \cdot\left[\rho^{*} \boldsymbol{u}^{*}\left(\frac{\epsilon_{0}}{u_{0}^{2}} \epsilon^{*}+\frac{u^{* 2}}{2}\right)+p^{*} \boldsymbol{u}^{*}\right]
\end{aligned}
$$

Again, we require this to be invariant under the scaling transformation, which leads to $\epsilon_{0} \equiv u_{0}^{2}$.

This simple exercise has shown that the equations of ideal MHD are indeed invariant under scaling. This applies for any choice of the scaling transformation. In reality, the case is more complex because neither the laboratory system nor the astrophysical one can be assumed to always evolve under ideal conditions. To see this, we consider Equation (1e) with the inclusion of the resistive, baroclinic, and Nernst terms. By applying the transformation defined above, with the additional inclusion of a temperature scaling $T \rightarrow T_{0} T^{*}$, we have

$$
\begin{aligned}
\frac{u_{0}}{\ell_{0}} & \sqrt{\mu_{0} \rho_{0}} u_{0} \frac{\partial \boldsymbol{B}^{*}}{\partial t^{*}}=-\frac{u_{0}}{\ell_{0}} \sqrt{\mu_{0} \rho_{0}} u_{0} \nabla^{*} \times\left(\boldsymbol{u}^{*} \times \boldsymbol{B}^{*}\right) \\
& +\eta \frac{u_{0}}{\ell_{0}^{2}} \sqrt{\mu_{0} \rho_{0}} u_{0} \nabla^{* 2} \boldsymbol{B}^{*}+\frac{m u_{0}^{2}}{e \ell_{0}^{2}(1+Z)} \frac{\nabla^{*} p^{*} \times \nabla^{*} \rho^{*}}{\rho^{* 2}} \\
& +\frac{\sqrt{\mu_{0} \rho_{0}} u_{0} T_{0}}{\ell_{0}^{2}} \frac{\tau_{e i}}{m_{e}} \frac{\beta_{1}^{\prime \prime} \chi^{2}+\beta_{0}^{\prime \prime}}{\Delta} \nabla^{*} \times\left(\boldsymbol{B}^{*} \times \nabla^{*} T^{*}\right) .
\end{aligned}
$$

Dividing through by $u_{0}^{2} \sqrt{\mu_{0} \rho_{0}} / \ell_{0}$,

$$
\begin{aligned}
\frac{\partial \boldsymbol{B}^{*}}{\partial t^{*}}= & \nabla^{*} \times\left(\boldsymbol{u}^{*} \times \boldsymbol{B}^{*}\right)+\frac{1}{\operatorname{Re}_{M}} \nabla^{* 2} \boldsymbol{B}^{*} \\
& +\frac{1}{B i} \frac{\nabla^{*} p^{*} \times \nabla^{*} \rho^{*}}{\rho^{* 2}}+\frac{1}{N e} \nabla^{*} \times\left(\boldsymbol{B}^{*} \times \nabla^{*} T^{*}\right),
\end{aligned}
$$

where can recognize the magnetic Reynolds number as

$$
R e_{M}=\frac{u_{0} \ell_{0}}{\eta},
$$

which represents the ratio of magnetic advection to magnetic diffusion, and the dimensionless numbers

$$
B i=\frac{e \sqrt{\mu_{0} \rho_{0}} \ell_{0}(1+Z)}{m}, \quad N e=\frac{u_{0} \ell_{0} m_{e}}{T_{0} \tau_{e i}} \frac{\Delta}{\beta_{1}^{\prime \prime} \chi^{2}+\beta_{0}^{\prime \prime}},
$$

which we will refer to as the Biermann number and Nernst number, respectively. These numbers represent the importance of magnetic field generation, due to the presence of electron currents, compared to magnetic field advection.

This shows that the equations of resistive MHD are scale invariant only if $R_{M}, B i$, and $\mathrm{Ne}$ are the same in both the laboratory and astrophysical systems or, alternatively, very large in both systems, such that these terms are negligible.

\section{SIMILARITY FOR NONIDEAL EQUATIONS IN THE OPTICALLY THICK CASE}

We must now consider the full system of Equations (1c) and (1d). In order to proceed, we need to define additional scaling variables for current density, electric field, and charge:

$$
\boldsymbol{J} \rightarrow J_{0} \boldsymbol{J}^{*}, \quad \boldsymbol{E} \rightarrow E_{0} \boldsymbol{E}^{*}, \quad \rho_{C} \rightarrow \rho_{C_{0}} \rho_{C}^{*} .
$$

\subsection{Momentum Equation}

We start with the momentum Equation (1b) and use the above scaling transformations by dividing through a common factor $\rho_{0} u_{0}^{2} / \ell_{0}$. After manipulation (for a more detailed derivation please see Appendix A) we get

$$
\begin{aligned}
& \rho^{*}\left(\frac{\partial \boldsymbol{u}^{*}}{\partial t^{*}}+\boldsymbol{u}^{*} \cdot \nabla^{*} \boldsymbol{u}^{*}\right) \\
& \quad=-\nabla^{*}\left[p^{*}+\frac{1}{R} T^{* 4}\right]+\frac{1}{\mathcal{H}_{Q}} \rho^{*} \nabla^{*}\left(\frac{\nabla^{* 2} \sqrt{\rho^{*}}}{\sqrt{\rho^{*}}}\right)
\end{aligned}
$$




$$
\begin{aligned}
& +\nabla^{*} \cdot\left\{\frac{1}{\operatorname{Re}}\left[\nabla^{*} \boldsymbol{u}^{*}+\left(\nabla^{*} \boldsymbol{u}^{*}\right)^{T}-\frac{2}{3}\left(\nabla^{*} \cdot \boldsymbol{u}^{*}\right) \underline{\boldsymbol{I}}\right]\right. \\
& \left.+\frac{1}{\operatorname{Re}_{\zeta}}\left(\nabla^{*} \cdot \boldsymbol{u}^{*}\right) \underline{\boldsymbol{I}}\right\}+\frac{1}{\Omega_{R}} \rho_{C}^{*} \boldsymbol{E}^{*}+\frac{1}{\Omega_{H}} \boldsymbol{J}^{*} \times \boldsymbol{B}^{*}
\end{aligned}
$$

The Mihalas number $(R)$ represents the ratio of ram pressure to radiation pressure, and it is related to the more familiar Boltzmann $(\mathrm{Bo})$ number by

$$
R=\frac{\rho_{0} u_{0}^{2}}{4 \sigma T_{0}^{4} / 3 c}=\frac{3 c}{4 u_{0}} \frac{\gamma-1}{\gamma} B o
$$

where $B o=\rho_{0} c_{p} T_{0} u_{0} / \sigma T_{0}^{4}$. Here, we have used $k_{B} T_{0} \sim m u_{0}^{2}$ and $c_{p} \sim \gamma k_{B} / m(\gamma-1)$. The Boltzmann number gives the ratio of the enthalpy flux to the radiation flux.

The importance of quantum effects to classical ones within the system is described by the number

$$
\mathcal{H}_{Q}=\frac{2 m_{e} m_{i} \ell_{0}^{2} u_{0}^{2}}{\hbar^{2}}
$$

which we will refer to as the Bohm number. We can also recognize the Reynold's number, the ratio of viscous to inertial effects, and its obvious extension when considering the second coefficient of viscosity:

$$
R e=\frac{\rho_{0} u_{0} \ell_{0}}{\mu} ; \quad R e_{\zeta}=\frac{\rho_{0} u_{0} \ell_{0}}{\zeta} .
$$

From charge conservation, $\rho_{C_{0}}=J_{0} / u_{0}$, it follows that

$$
\Omega_{R}=\frac{\rho_{0} u_{0}^{2}}{\rho_{C_{0}} \ell_{0} E_{0}}=\frac{\rho_{0} u_{0}^{3}}{J_{0} E_{0} \ell_{0}},
$$

which represents the ratio between Ohmic and convective heat transfer, which we call the ohmic number. The ratio between convective transport and Hall diffusion is expressed by the coefficient

$$
\Omega_{H}=\frac{u_{0} \sqrt{\mu_{0} \rho_{0}}}{J_{0} \ell_{0}}=\frac{\mu_{0} \rho_{0} u_{0}^{2}}{J_{0} B_{0} \ell_{0}},
$$

which we refer to as the Hall number.

\subsection{Energy Equation}

Following the same approach as before, but now using the energy Equation (1c) and dividing through by a common factor of $\rho_{0} u_{0}^{3} / \ell_{0}$, the dimensionless energy equation can thus be written as (see Appendix B)

$$
\begin{aligned}
\frac{\partial}{\partial t^{*}}\left(\rho^{*} \epsilon^{*}+\frac{\rho^{*} u^{* 2}}{2}+\frac{3}{R} T^{* 4}\right)+\nabla^{*} \cdot\left[\rho^{*} \boldsymbol{u}^{*}\left(\epsilon^{*}+\frac{u^{2}}{2}\right)+p^{*} \boldsymbol{u}^{*}\right] \\
=\nabla^{*} \cdot\left\{\frac{1}{\Pi_{\mathrm{thick}}} \frac{T^{* 3}}{\rho^{*}} \nabla^{*} T^{*}-\frac{3}{R} T^{* 4} \boldsymbol{u}^{*}+\frac{1}{P e} \frac{\gamma}{\gamma-1} \rho^{*} \nabla^{*} T^{*}\right. \\
\quad+\frac{1}{\operatorname{Re}}\left[\nabla^{*} \boldsymbol{u}^{*}+\left(\nabla^{*} \boldsymbol{u}^{*}\right)^{T}-\frac{2}{3}\left(\nabla^{*} \cdot \boldsymbol{u}^{*}\right) \underline{\boldsymbol{I}}\right] \cdot \boldsymbol{u}^{*} \\
\left.\quad+\frac{1}{\operatorname{Re}_{\zeta}}\left(\nabla^{*} \cdot \boldsymbol{u}^{*}\right) \underline{\boldsymbol{I}} \cdot \boldsymbol{u}^{*}\right\}-\frac{1}{\Omega_{R}} \boldsymbol{J}^{*} \cdot \boldsymbol{E}^{*} \\
\quad+\frac{1}{\mathcal{H}_{Q}} \rho^{*} \nabla^{*}\left(\frac{\nabla^{* 2} \sqrt{\rho^{*}}}{\sqrt{\rho^{*}}}\right) \cdot \boldsymbol{u}^{*}-\frac{1}{R} T^{* 4} \nabla^{*} \cdot \boldsymbol{u}^{*} .
\end{aligned}
$$

Analogous to the momentum equation, we have new dimensionless numbers. We define the radiation number, $\Pi_{\text {thick }}$, which is related to the Boltzmann number (in the same way as the Mihalas number, above) by

$$
\Pi_{\text {thick }}=\frac{3 \kappa_{R} \rho_{0}^{2} \ell_{0} u_{0}^{3}}{16 \sigma T_{0}^{4}}\left(=\frac{3 \ell_{0}}{16 \lambda_{R}} \frac{\gamma-1}{\gamma} B o\right) .
$$

This number describes the importance of material energy flux compared to the radiative energy flux, weighted by the ratio of the mean free path of the radiation, $\lambda_{R}=1 / \kappa_{R} \rho_{0}$, to the characteristic length scale of the system. The Péclet number gives the importance of thermal diffusion against convective transport:

$$
P e=\frac{\ell_{0} m u_{0}^{3}}{\chi_{\mathrm{th}} k_{B} T_{0}}=\frac{\ell_{0} u_{0}}{\chi_{\mathrm{th}}},
$$

where we have used again the relation $k_{B} T \sim m u_{0}^{2}$.

\section{SIMILARITY FOR NONIDEAL EQUATIONS IN THE OPTICALLY THIN CASE}

It is worth noting that the Mihalas and radiation numbers as shown above rely on the material in question being optically thick to radiation. The form of the equations as formulated so far cannot be used in the presence of an optically thin plasma. The scaling relations in the optically thin case have been discussed in terms of cooling functions and characteristic timescales (Ryutov et al. 1999, 2001) and using Lie group theory (Falize et al. 2011b). Moreover, in the special situation of thick-thin radiation transport a more complex treatment is required (McClarren et al. 2010).

Under optically thin conditions, the source terms relating to radiation can be written as

$$
\begin{gathered}
p_{R}=0 \\
f_{\mathrm{rad}}=0,
\end{gathered}
$$

where the transfer of momentum to the plasma by radiation is zero, by definition, because the plasma is optically thin, and the remaining radiation terms, relating to radiative energy flux (Equation (19)), are written in terms of a cooling function

$$
L_{\Lambda}=\frac{\partial E_{R}}{\partial t}+\nabla \cdot\left[\boldsymbol{F}_{R}+E_{R} \boldsymbol{u}\right] \approx \rho \kappa_{P} \sigma T^{4} .
$$

This can be approximated with a form that is similar to the optically thick case (Equation (2b)), where $\kappa_{P}$ is the Planck opacity (Falize et al. 2011b).

The dimensionless momentum and energy equations now read as follows.

\subsection{Momentum Equation}

$$
\begin{aligned}
\rho^{*} & \left(\frac{\partial \boldsymbol{u}^{*}}{\partial t^{*}}+\boldsymbol{u}^{*} \cdot \nabla^{*} \boldsymbol{u}^{*}\right)=-\nabla^{*} p^{*}+\frac{1}{\mathcal{H}_{Q}} \rho^{*} \nabla^{*}\left(\frac{\nabla^{* 2} \sqrt{\rho^{*}}}{\sqrt{\rho^{*}}}\right) \\
& +\nabla^{*} \cdot\left\{\frac{1}{\operatorname{Re}}\left[\nabla^{*} \boldsymbol{u}^{*}+\left(\nabla^{*} \boldsymbol{u}^{*}\right)^{T}-\frac{2}{3}\left(\nabla^{*} \cdot \boldsymbol{u}^{*}\right) \underline{\boldsymbol{I}}\right]\right. \\
& \left.+\frac{1}{R e_{\zeta}}\left(\nabla^{*} \cdot \boldsymbol{u}^{*}\right) \underline{\boldsymbol{I}}\right\}+\frac{1}{\Omega_{R}} \rho_{C}^{*} \boldsymbol{E}^{*}+\frac{1}{\Omega_{H}} \boldsymbol{J}^{*} \times \boldsymbol{B}^{*}
\end{aligned}
$$


Table 2

List of Scaling Variables and Dimensionless Numbers

\begin{tabular}{|c|c|}
\hline Characteristic Quantity & Definition \\
\hline Length & $\ell_{0}$ \\
\hline Velocity & $u_{0}$ \\
\hline Density & $\rho_{0}$ \\
\hline Current density & $J_{0}$ \\
\hline Electric field & $E_{0}$ \\
\hline Temperature & $T_{0}$ \\
\hline Time & $t_{0}=\ell_{0} / u_{0}$ \\
\hline Pressure & $p_{0}=\rho_{0} u_{0}^{2}$ \\
\hline Magnetic field & $B_{0}=u_{0} \sqrt{\mu_{0} \rho_{0}}$ \\
\hline Specific internal energy & $\epsilon_{0}=u_{0}^{2}$ \\
\hline Charge density & $\rho_{C_{0}}=J_{0} / u_{0}$ \\
\hline Reynolds number & $R e=\rho_{0} u_{0} \ell_{0} / \mu$ \\
\hline Reynolds number (bulk) & $R e_{\zeta}=\rho_{0} u_{0} \ell_{0} / \zeta$ \\
\hline Magnetic Reynolds number & $\operatorname{Re}_{M}=u_{0} \ell_{0} / \eta$ \\
\hline Biermann number & $B i=e(1+Z) \sqrt{\mu_{0} \rho_{0}} \ell_{0} / m$ \\
\hline Nernst number & $N e=u_{0} \ell_{0} m_{e} \Delta / T_{0} \tau_{e i}\left(\beta_{1}^{\prime \prime} \chi^{2}+\beta_{0}^{\prime \prime}\right)$ \\
\hline Mihalas number & $R=3 c \rho_{0} u_{0}^{2} / 4 \sigma T_{0}^{4}$ \\
\hline Radiation number (Thick) & $\Pi_{\text {thick }}=3 \ell_{0} \rho_{0} u_{0}^{3} / 16 \lambda_{R} \sigma T_{0}^{4}$ \\
\hline Radiation number (Thin) & $\Pi_{\text {thin }}=\lambda_{P} \rho_{0} u_{0}^{3} / \ell_{0} \sigma T_{0}^{4}$ \\
\hline Péclet number & $P e=\ell_{0} u_{0} / \chi_{t h}$ \\
\hline Ohmic number & $\Omega_{R}=\rho_{0} u_{0}^{3} / J_{0} E_{0} \ell_{0}$ \\
\hline Hall number & $\Omega_{H}=\mu_{0} \rho_{0} u_{0}^{2} / J_{0} B_{0} \ell_{0}$ \\
\hline Bohm number & $\mathcal{H}_{Q}=2 m_{e} m_{i} u_{0}^{2} \ell_{0}^{2} / \hbar^{2}$ \\
\hline
\end{tabular}

\subsection{Energy Equation}

$$
\begin{aligned}
& \frac{\partial}{\partial t^{*}}\left(\rho^{*} \epsilon^{*}+\frac{\rho^{*} u^{* 2}}{2}\right)+\nabla^{*} \cdot\left[\rho^{*} \boldsymbol{u}^{*}\left(\epsilon^{*}+\frac{u^{2}}{2}\right)+p^{*} \boldsymbol{u}^{*}\right]=\nabla^{*} \\
& \cdot\left\{\frac{1}{P e} \frac{\gamma}{\gamma-1} \rho^{*} \nabla^{*} T^{*}+\frac{1}{\operatorname{Re}}\left[\nabla^{*} \boldsymbol{u}^{*}+\left(\nabla^{*} \boldsymbol{u}^{*}\right)^{T}-\frac{2}{3}\left(\nabla^{*} \cdot \boldsymbol{u}^{*}\right) \underline{\boldsymbol{I}}\right]\right. \\
& \left.\cdot \boldsymbol{u}^{*}+\frac{1}{R e_{\zeta}}\left(\nabla^{*} \cdot \boldsymbol{u}^{*}\right) \underline{\boldsymbol{I}} \cdot \boldsymbol{u}^{*}\right\}-\frac{1}{\Omega_{R}} \boldsymbol{J}^{*} \cdot \boldsymbol{E}^{*} \\
& +\frac{1}{\mathcal{H}_{Q}} \rho^{*} \nabla^{*}\left(\frac{\nabla^{* 2} \sqrt{\rho^{*}}}{\sqrt{\rho^{*}}}\right) \cdot \boldsymbol{u}^{*}-\frac{1}{\Pi_{\text {thin }}} \rho^{*} T^{* 4}
\end{aligned}
$$

As for the optically thick case, we recover a set of dimensionless characteristic numbers. The only difference is that the radiation term is now altered, and the proper number to use in this case is

$$
\Pi_{\text {thin }}=\frac{u_{0}^{3}}{\kappa_{p} \sigma \ell_{0} T_{0}^{4}}=\frac{\lambda_{P}}{\ell_{0}} \frac{\rho_{0} u_{0}^{3}}{\sigma T_{0}^{4}}\left(=\frac{\lambda_{P}}{\ell_{0}} \frac{\gamma-1}{\gamma} B o\right),
$$

which has a similar form to the radiation number for the optically thick case. It is a measure of the ratio between the material and radiative energy fluxes, weighted by the ratio of the mean free path, $\lambda_{P}=1 / \kappa_{P} \rho_{0}$, to the characteristic length scale of the system $\ell_{0}$. However, please note that the ratio between the radiation mean free path and $\ell_{0}$ is reversed when going from the optically thick to the optically thin regime.

\section{DISCUSSION}

A summary of the scaling variables and all of the dimensionless number is given in Table 2. As discussed earlier, similarity between the laboratory and astrophysical object is achieved if the dimensionless numbers are the same or sufficiently large in both systems (the ideal MHD case). Under either of these conditions, we take $\ell_{0}^{(1)}, u_{0}^{(1)}, \rho_{0}^{(1)}, J_{0}^{(1)}, E_{0}^{(1)}$, and $T_{0}^{(1)}$ as the characteristic scaling parameters for the laboratory experiment. The astrophysical system has corresponding values given by

$$
\begin{aligned}
& \ell_{0}^{(2)}=g_{a} \ell_{0}^{(1)}, \quad u_{0}^{(2)}=g_{b} u_{0}^{(1)}, \quad \rho_{0}^{(2)}=g_{c} \rho_{0}^{(1)}, \\
& J_{0}^{(2)}=g_{d} J_{0}^{(1)}, \quad E_{0}^{(2)}=g_{e} E_{0}^{(1)}, \quad T_{0}^{(2)}=g_{f} T_{0}^{(1)},
\end{aligned}
$$

where $g_{a, b, c, d, e, f}$ are scaling constants. From this set of parameters, we can scale all of the other characteristic quantities as

$$
\begin{aligned}
t_{0}^{(2)} & =\frac{g_{a}}{g_{b}} t_{0}^{(1)}, \quad p_{0}^{(2)}=g_{c} g_{b}^{2} p_{0}^{(1)}, \quad B_{0}^{(2)}=g_{b} \sqrt{g_{c}} B_{0}^{(1)}, \\
\epsilon_{0}^{(2)} & =g_{b}^{2} \epsilon_{0}^{(1)}, \quad \rho_{C_{0}}^{(2)}=\frac{g_{d}}{g_{b}} \rho_{C_{0}}^{(1)} .
\end{aligned}
$$

All of the details concerning the microphysics of the two systems are thus contained only in the dimensionless numbers given in Table 2. In order to evaluate these numbers, let us assume the plasma is in thermodynamic equilibrium at temperature $T$ (in $\mathrm{eV})$ and carries a mass density $\rho\left(\mathrm{in} \mathrm{g} / \mathrm{cm}^{3}\right)$ from ions of atomic mass $A$ and charge $Z$. The magnetic field is $B$ (in $G$ ). Charge neutrality implies an equal number of negative charges carried by mobile electrons. These assumptions are applicable to both the laboratory and astrophysical plasmas. Following Ryutov et al. (1999) and Huba (2002), the kinematic viscosity is

$$
v\left(\mathrm{~cm}^{2} \mathrm{~s}^{-1}\right)=\operatorname{Min}\left\{\begin{array}{l}
3.3 \times 10^{-5} \frac{A^{1 / 2} T^{5 / 2}}{Z^{4} \rho \Lambda} \\
2.8 \times 10^{43} \frac{\rho^{2} \Lambda}{A^{5 / 2} Z^{2} B^{2} T^{1 / 2}}
\end{array}\right\},
$$

where $\Lambda$ is the Coulomb logarithm. The thermal diffusivity is (Ryutov et al. 1999)

$$
\chi_{\mathrm{th}}\left(\mathrm{cm}^{2} \mathrm{~s}^{-1}\right)=\operatorname{Min}\left\{\begin{array}{l}
3.3 \times 10^{-3} \frac{A T^{5 / 2}}{Z(Z+1) \rho \Lambda} \\
8.6 \times 10^{9} \frac{A^{1 / 2} T}{Z B}
\end{array}\right\} .
$$

The magnetic diffusivity can be written as (Lifshitz \& Pitaevskii 1981)

$$
\eta\left(\mathrm{cm}^{2} \mathrm{~s}^{-1}\right)=2.4 \times 10^{5} \frac{Z \Lambda}{T^{3 / 2}} .
$$

The electron-ion collision time is given by (Huba 2002)

$$
\tau_{e i}(\mathrm{~s})=5.2 \times 10^{-16} \frac{A^{2} T^{3 / 2}}{Z^{2} \rho \Lambda} .
$$

In the case of a fully ionized plasma, the Rosseland opacity is only determined by the free-free absorption, thus (Zeldovich et al. 1966)

$$
\kappa_{R}\left(\mathrm{~cm}^{2} \mathrm{~g}^{-1}\right)=4.4 \times 10^{8} \frac{Z^{3} \rho}{A^{2} T^{7 / 2}} .
$$

For typical astrophysical plasmas, the Planck opacity is (Sutherland \& Dopita 1993)

$$
\kappa_{P}\left(\mathrm{~cm}^{2} \mathrm{~g}^{-1}\right)=1.8 \times 10^{13} \frac{Z \rho}{A^{2} T^{4}},
$$

and for bremsstrahlung-dominated cooling (Ryutov et al. 1999)

$$
\kappa_{P}\left(\mathrm{~cm}^{2} \mathrm{~g}^{-1}\right)=3.1 \times 10^{10} \frac{Z^{2} \rho}{A^{2} T^{7 / 2}} .
$$

At higher densities (near and above solid) and when line radiation transport must be included in the calculations, the 
Table 3

List of Coefficient Values for Rosseland Opacity from Equation (29)

\begin{tabular}{lccc}
\hline \hline Material & $\kappa_{0}$ & $\alpha$ & $\beta$ \\
\hline $\mathrm{CH}$ & $2.00 \times 10^{6}$ & 0.14 & -2.00 \\
$\mathrm{Al}$ & $1.04 \times 10^{8}$ & 0.48 & -2.48 \\
$\mathrm{Ti}$ & $3.07 \times 10^{7}$ & 0.39 & -2.21 \\
$\mathrm{Fe}$ & $6.29 \times 10^{7}$ & 0.31 & -2.27 \\
$\mathrm{Cu}$ & $5.93 \times 10^{7}$ & 0.29 & -2.21 \\
$\mathrm{Mo}$ & $1.99 \times 10^{6}$ & 0.22 & -1.49 \\
$\mathrm{Sn}$ & $3.70 \times 10^{6}$ & 0.16 & -1.57 \\
$\mathrm{Xe}$ & $2.00 \times 10^{8}$ & 0.00 & -2.00 \\
$\mathrm{Ba}$ & $5.89 \times 10^{6}$ & 0.14 & -1.62 \\
$\mathrm{Eu}$ & $2.89 \times 10^{6}$ & 0.09 & -1.45 \\
$\mathrm{~W}$ & $5.59 \times 10^{5}$ & 0.01 & -1.12 \\
$\mathrm{Au}$ & $6.00 \times 10^{6}$ & 0.30 & -1.50 \\
$\mathrm{~Pb}$ & $4.11 \times 10^{5}$ & 0.00 & -1.05 \\
$\mathrm{U}$ & $7.76 \times 10^{5}$ & 0.04 & -1.14 \\
\hline
\end{tabular}

Note. Adapted from Tsakiris \& Eidmann (1987) and Drake (2006).

Table 4

List of Coefficient Values for Planck Opacity from Equation (29)

\begin{tabular}{lccc}
\hline \hline Material & $\kappa_{0}$ & $\alpha$ & $\beta$ \\
\hline $\mathrm{CH}$ & $2.00 \times 10^{5}$ & 0.00 & -1.00 \\
$\mathrm{Al}$ & $6.01 \times 10^{8}$ & 0.48 & -2.42 \\
$\mathrm{Ti}$ & $1.40 \times 10^{8}$ & 0.44 & -2.07 \\
$\mathrm{Fe}$ & $2.22 \times 10^{8}$ & 0.38 & -2.13 \\
$\mathrm{Cu}$ & $2.31 \times 10^{8}$ & 0.36 & -2.22 \\
$\mathrm{Mo}$ & $1.54 \times 10^{7}$ & 0.31 & -1.56 \\
$\mathrm{Sn}$ & $1.91 \times 10^{7}$ & 0.23 & -1.59 \\
$\mathrm{Xe}$ & $3.00 \times 10^{9}$ & 0.00 & -2.00 \\
$\mathrm{Ba}$ & $2.77 \times 10^{7}$ & 0.24 & -1.64 \\
$\mathrm{Eu}$ & $1.68 \times 10^{7}$ & 0.24 & -1.54 \\
$\mathrm{~W}$ & $3.06 \times 10^{6}$ & 0.20 & -1.23 \\
$\mathrm{Au}$ & $3.33 \times 10^{6}$ & 0.17 & -1.23 \\
$\mathrm{~Pb}$ & $4.17 \times 10^{6}$ & 0.16 & -1.27 \\
$\mathrm{U}$ & $1.04 \times 10^{7}$ & 0.19 & -1.42 \\
\hline
\end{tabular}

Note. Adapted from Tsakiris \& Eidmann (1987) and Drake (2006).

Rosseland and Planck opacities are tabulated as (Tsakiris \& Eidmann 1987)

$$
\kappa_{P, R}\left(\mathrm{~cm}^{2} \mathrm{~g}^{-1}\right)=\kappa_{0} \rho^{\alpha} T^{\beta},
$$

where $\kappa_{0}, \alpha$, and $\beta$ are material-dependent constants (see Tables 3 and 4). The Rosseland and Planck opacities are bound to a maximum value given by (Tsakiris \& Eidmann 1987)

$$
\kappa_{P, R}^{\max }\left(\mathrm{cm}^{2} \mathrm{~g}^{-1}\right)=6.1 \times 10^{6} \frac{Z}{A T} .
$$

Even in the case that the dimensionless numbers are large in both the laboratory and astrophysical systems, their magnitude can be very different. It is then important to quantify the error in fluid variables in the ideal MHD approximation due to finite values for such dimensionless numbers. For the optically thick case, we have

$$
\begin{gathered}
\frac{\Delta B}{B_{\mathrm{id}}} \sim\left(\frac{1}{R e_{M}^{2}}+\frac{1}{B i^{2}}+\frac{1}{N e^{2}}\right)^{1 / 2}, \\
\frac{\Delta \rho u}{(\rho u)_{\mathrm{id}}} \sim\left(\frac{1}{R^{2}}+\frac{1}{\mathcal{H}_{Q}^{2}}+\frac{1}{R e^{2}}+\frac{1}{R e_{\zeta}^{2}}+\frac{1}{\Omega_{R}^{2}}+\frac{1}{\Omega_{H}^{2}}\right)^{1 / 2},
\end{gathered}
$$

Table 5

Example of Scaling under Radiative Conditions from the Laboratory (Pak et al. 2013) to a Supernova Shock Breakout

\begin{tabular}{lcc}
\hline \hline Characteristic Quantity & Lab & Astro \\
\hline Length & $100 \mu \mathrm{m}$ & $1.0 \times 10^{8} \mathrm{~km}$ \\
Velocity & $300 \mathrm{~km} \mathrm{~s}^{-1}$ & $3.0 \times 10^{3} \mathrm{~km} \mathrm{~s}^{-1}$ \\
Density & $1 \mathrm{~g} \mathrm{~cm}^{-3}$ & $1.7 \times 10^{-9} \mathrm{~g} \mathrm{~cm}^{-3}$ \\
Temperature & $250 \mathrm{eV}$ & $1000 \mathrm{eV}$ \\
\hline Time & $300 \mathrm{ps}$ & $9 \mathrm{hr}$ \\
Pressure (Ram) & $90 \mathrm{TPa}$ & $15 \mathrm{MPa}$ \\
\hline Reynolds number & $4.0 \times 10^{6}$ & $1.9 \times 10^{12}$ \\
Magnetic Reynolds number & 8.7 & $1.0 \times 10^{20}$ \\
Biermann number & 130 & $5.2 \times 10^{13}$ \\
Nernst number & 0.1 & $1.3 \times 10^{6}$ \\
Mihalas number & 5,000 & $3.3 \times 10^{-6}$ \\
Radiation number (Thick) & 0.5 & $2.7 \times 10^{-4}$ \\
Péclet number & 1,200 & $3.6 \times 10^{9}$ \\
Bohm number & $6.4 \times 10^{19}$ & $2.5 \times 10^{48}$
\end{tabular}

$$
\frac{\Delta \rho \epsilon}{(\rho \epsilon)_{\mathrm{id}}} \sim\left(\frac{1}{R^{2}}+\frac{1}{\Pi_{\text {thick }}^{2}}+\frac{1}{P e^{2}}+\frac{1}{\mathcal{H}_{Q}^{2}}+\frac{1}{R e^{2}}+\frac{1}{R e_{\zeta}^{2}}+\frac{1}{\Omega_{R}^{2}}\right)^{1 / 2}
$$

where $B_{\text {id }},(\rho u)_{\text {id }}$, and $(\rho \epsilon)_{\text {id }}$ refer to the magnetic field, momentum, and energy, respectively, in the ideal MHD approximation. Similar relations can be straightforwardly derived for optically thin plasmas.

\section{EXPERIMENTAL COMPARISON}

In this section we apply the scaling relations to a few recent experiments and discuss how they can be used to meaningfully describe astrophysical environments. We focus our attention to the case when radiation becomes important, mainly because, as we will see below, this is where similarity between the laboratory and the astrophysical systems is difficult to achieve. By contrast, in the absence of significant radiative effects, hydrodynamic or MHD similarities have been successfully applied to a wide range of problems. A comprehensive review of laboratory astrophysics experiments is given by Remington et al. (1999, 2006); Drake (2006); Savin et al. (2012).

First, we consider a recent implosion experiment on the National Ignition Facility (NIF) laser (Pak et al. 2013), which is related to shock waves in core-collapse supernova explosions surrounded by an optically thick envelope or wind. Typical values for these types of systems are given, based on values from Katz et al. 2010, in Table 5. Although the experiment can indeed reproduce the supernova shock in many aspects, the similarity breaks down when considering the Mihalas number and is only marginally satisfied for magnetic transport (because of diffusion and advection along the temperature gradient rather than with the fluid flow, as in the astrophysical case). This means that the radiation pressure is significantly smaller than the material pressure in the laboratory, and it does not change the form of the energy equation, unlike the astrophysical case where radiation pressure is much more important than the material pressure. Even if $R \gg 1$ in the laboratory, the effect of radiation can be important due to radiation flux in the energy equation, but in this case, the large difference in the radiation number makes the similarity marginally satisfied. This example shows that radiation-dominated environments are still challenging to achieve even on the currently available largest laser facilities. 
Table 6

Comparison of Laboratory Experiment to an Astrophysical Case (Herbig-Haro Object)

\begin{tabular}{lcc}
\hline \hline Characteristic Quantity & Lab & Astro \\
\hline Length & $150 \mu \mathrm{m}$ & $1.4 \times 10^{6} \mathrm{~km}$ \\
Velocity & $500 \mathrm{~km} \mathrm{~s}^{-1}$ & $35 \mathrm{~km} \mathrm{~s}^{-1}$ \\
Density & $1 \times 10^{-4} \mathrm{~g} \mathrm{~cm}^{-3}$ & $6.6 \times 10^{-10} \mathrm{~g} \mathrm{~cm}^{-3}$ \\
Temperature & $100 \mathrm{eV}$ & $15 \mathrm{eV}$ \\
\hline Time & $300 \mathrm{ps}$ & $0.5 \mathrm{days}$ \\
Pressure (Ram) & $25 \mathrm{GPa}$ & $810 \mathrm{~Pa}$ \\
\hline Reynolds number & 1800 & $1.8 \times 10^{7}$ \\
Magnetic Reynolds number & 160 & $1.56 \times 10^{16}$ \\
Biermann number & 3.8 & $1.9 \times 10^{9}$ \\
Nernst number & $1.9 \times 10^{-4}$ & 12 \\
Radiation number (Thin) & 460 & $4.4 \times 10^{-6}$ \\
Péclet number & 3.4 & $3.4 \times 10^{4}$ \\
Bohm number & $6.2 \times 10^{15}$ & $6.2 \times 10^{49}$ \\
\hline
\end{tabular}

Note. From Tikhonchuk et al. (2008).

Radiative jets and outflows are present in several young stellar objects (Reipurth \& Bally 2001). Among more recent work, we focus on the experiment by Tikhonchuk et al. (2008), who claim to have good scaling between their experiment and astrophysics and have entered a regime where radiative effects are important. The scaling relations and corresponding dimensionless numbers are given in Table 6 . We indeed see that in this specific case, the experiment succeeds in matching the trend, if not magnitude, of many of the dimensionless numbers. However, the radiation number remains too large compared to the astrophysical case: the material energy flux in the laboratory is dominating over the radiation flux. Similarity is partially satisfied when considering magnetic Reynolds and Biermann numbers, suggesting that the magnetic field diffusion is negligible, but the difference in the Nernst number indicates the importance of thermal advection in the laboratory case.

Other experiments have been able to better match values relating to radiative effects. In the experiments by Krauland et al. (2013a, 2013b) the values of $\Pi_{\text {thin }}$ are similar in the experimental and astrophysical cases: $\sim 0.03$ and $\sim 1$, respectively. Thus the radiative and material energy fluxes are comparable within an order of magnitude. This is characteristic of the more complex optically thick postshock, optically thin preshock conditions found, for example, in accretion processes in interacting binary star systems.

It is also important to note that, although values of these characteristic ratios can lead to one process dominating over the other (e.g., the effects of radiative flux are greater than those from material flux in the laboratory; see Table 5), their ratio could be many orders of magnitude different from the astrophysical case. A well-scaled experiment would show, at least, the correct trend in the ratio of the characteristic values (i.e., large if the value for the astrophysical case is large, or vice versa) for all quantities.

Another aspect of the scaling relations that is worth discussing is the importance of the Bohm potential. Although this term is of no significance in the tenuous interstellar plasma, it can become important when considering small scales or compact objects, particularly for densities exceeding $10^{23}-10^{29} \mathrm{~cm}^{-3}$ (Haas 2011), which are found, for example, in white dwarfs and neutron stars.

This is particularly relevant when considering, for example, Kolmogorov turbulence (Kolmogorov 1991). In the inertial
Table 7

Typical Parameters for White Dwarf Stars, Adapted from Lai (2001) and Zingale et al. (2009)

\begin{tabular}{lc}
\hline \hline Characteristic Quantity & Astro \\
\hline Length & $10^{3} \mathrm{~km}$ \\
Velocity & $50 \mathrm{~km} \mathrm{~s}^{-1}$ \\
Density & $10^{7} \mathrm{~g} \mathrm{~cm}^{-3}$ \\
Temperature & $10 \mathrm{keV}$ \\
\hline$\ell_{q} / \ell_{v}$ & 50
\end{tabular}

range $\rho u_{\ell}^{3} / \ell=\dot{\epsilon}=$ constant, where $u_{\ell}$ is the characteristic velocity at scale $\ell$, and $\dot{\epsilon}$ is the total power injected into turbulence. Hence the characteristic eddy turnover rate at scale $\ell$ is $u_{\ell} / \ell \sim(\dot{\epsilon} / \rho)^{1 / 3} \ell^{-2 / 3}$. Quantum effects are expected to become important when $\hbar^{2} / 2 m_{e} \ell^{2} \sim m u_{\ell}^{2}$, which defines the scale

$$
\ell_{q} \simeq\left(\frac{\hbar^{2}}{2 m_{e} m}\right)^{3 / 8}\left(\frac{\rho}{\dot{\epsilon}}\right)^{1 / 4}
$$

We also notice that the rate of viscous dissipation on a scale $\ell$ is given by $v / \ell^{2}$. Equating this to the eddy turnover rate, we determine the scale at which viscous dissipation becomes dominant:

$$
\ell_{v} \simeq v^{3 / 4}\left(\frac{\rho}{\dot{\epsilon}}\right)^{1 / 4}
$$

and quantum effects will lie within the inertial range if $\ell_{q}>\ell_{\nu}$, or

$$
v\left(\mathrm{~cm}^{2} \mathrm{~s}^{-1}\right)<\frac{1.9 \times 10^{-4}}{A^{1 / 2}} .
$$

Because the viscosity decreases as a function of the density, it is then obvious to expect that quantum effects will become more important at higher densities. Similar considerations apply to the resistive scale. If the above conditions are satisfied, we would see some change in the structure of turbulence below the scale $\ell_{q}$. This can become important when considering the fluid core of white dwarf stars (Bildsten 2001), as shown in Table 7.

\section{CONCLUDING REMARKS}

In this paper we have provided a comprehensive description of the MHD scaling in the presence of quantum, resistive, and radiative effects. The dimensionless form of these equations reveals a set of characteristic numbers that can be used to quantify the departure from the ideal fluid behavior. The scale-invariance properties of the MHD equations have been successfully exploited to describe astrophysical phenomena in a variety of laboratory experiments (Remington et al. 1999; Drake 2006; Savin et al. 2012), and here we have provided a unified theoretical framework that is common to all of these experiments and can be applied to the planning and analysis of future ones.

The research leading to these results has received funding from the European Research Council under the European Community's Seventh Framework Programme (FP7/2007-2013) / ERC grant agreement No. 256973. Partial support from AWE plc is also acknowledged. The authors would like to thank the anonymous referee for important insights provided into the manuscript.

The derivation of the dimensionless form of the momentum and energy equations (in the optically thick regime) is outlined here in detail. 


\section{APPENDIX A}

\section{MOMENTUM EQUATION}

Considering each term separately, we have

$$
\begin{array}{r}
\rho\left(\frac{\partial \boldsymbol{u}}{\partial t}+\boldsymbol{u} \cdot \nabla \boldsymbol{u}\right) \rightarrow \frac{\rho_{0} u_{0}^{2}}{\ell_{0}} \rho^{*}\left(\frac{\partial \boldsymbol{u}^{*}}{\partial t^{*}}+\boldsymbol{u}^{*} \cdot \nabla^{*} \boldsymbol{u}^{*}\right) \quad \text { (A1a) } \\
-\nabla\left(p+\frac{4 \sigma T^{4}}{3 c}\right) \rightarrow-\frac{\rho_{0} u_{0}^{2}}{\ell_{0}} \nabla^{*}\left(p^{*}+\frac{4 \sigma T_{0}^{4}}{3 \rho_{0} u_{0}^{2} c} T^{* 4}\right) \quad(\mathrm{A} 1 \mathrm{~b}) \\
\frac{\hbar^{2} \rho}{2 m^{2}} \nabla\left(\frac{\nabla^{2} \sqrt{\rho}}{\sqrt{\rho}}\right) \rightarrow \frac{\rho_{0} u_{0}^{2}}{\ell_{0}}\left(\frac{\hbar}{\ell_{0} u_{0} \sqrt{2} m}\right)^{2} \rho^{*} \nabla^{*}\left(\frac{\nabla^{* 2} \sqrt{\rho^{*}}}{\sqrt{\rho^{*}}}\right) \\
\nabla \cdot\{\rho v[\nabla \boldsymbol{A} 1 \mathrm{c}) \\
\rightarrow \frac{\rho_{0} u_{0}^{2}}{\ell_{0}} \nabla^{*} \cdot\left\{\frac{\mu}{\rho_{0} u_{0} \ell_{0}}\left[\nabla^{*} \boldsymbol{u}^{*}+\left(\nabla^{*} \boldsymbol{u}^{*}\right)^{T}-\frac{2}{3}\left(\nabla^{*} \cdot \boldsymbol{u}^{*}\right) \underline{\boldsymbol{I}}\right]\right. \\
\left.+\frac{\zeta}{\rho_{0} u_{0} \ell_{0}}\left(\nabla^{*} \cdot \boldsymbol{u}^{*}\right) \underline{\boldsymbol{I}}\right\} \\
\rho_{C} \boldsymbol{E}+\boldsymbol{J} \times \boldsymbol{B} \rightarrow \frac{\rho_{0} u_{0}^{2}}{\ell_{0}}\left(\frac{\rho_{C_{0}} \ell_{0} E_{0}}{\rho_{0} u_{0}^{2}} \rho_{C}^{*} \boldsymbol{E}^{*}+\frac{J_{0} \ell_{0}}{u_{0} \sqrt{\mu_{0} \rho_{0}}} \boldsymbol{J}^{*} \times \boldsymbol{B}^{*}\right) .
\end{array}
$$

If we divide through by the common term $\rho_{0} u_{0}^{2} / \ell_{0}$, we obtain Equation (9).

\section{APPENDIX B}

\section{ENERGY EQUATION}

Considering again each term separately as we have done for the momentum equation

$$
\begin{gathered}
\frac{\partial}{\partial t}\left(\rho \epsilon+\frac{\rho u^{2}}{2}+\frac{4 \sigma T^{4}}{c}\right) \rightarrow \frac{\rho_{0} u_{0}^{3}}{\ell_{0}} \frac{\partial}{\partial t^{*}} \\
\times\left(\rho^{*} \epsilon^{*}+\frac{\rho^{*} u^{* 2}}{2}+\frac{4 \sigma T_{0}^{4}}{\rho_{0} u_{0}^{2} c} T^{* 4}\right), \\
\nabla \cdot\left[\rho \boldsymbol{u}\left(\epsilon+\frac{u^{2}}{2}\right)+p \boldsymbol{u}\right] \rightarrow \frac{\rho_{0} u_{0}^{3}}{\ell_{0}} \nabla^{*} \\
\cdot\left[\rho^{*} \boldsymbol{u}^{*}\left(\epsilon^{*}+\frac{u^{2}}{2}\right)+p^{*} \boldsymbol{u}^{*}\right], \\
\nabla \cdot\left(\frac{16 \sigma T^{3}}{3 \kappa_{R} \rho} \nabla T\right) \rightarrow \frac{\rho_{0} u_{0}^{3}}{\ell_{0}} \nabla^{*} \cdot\left(\frac{16 \sigma T_{0}^{4}}{3 \kappa_{R} \rho_{0}^{2} \ell_{0} u_{0}^{3}} \frac{T^{* 3}}{\rho^{*}} \nabla^{*} T^{*}\right), \\
-\nabla \cdot\left(\frac{3 \sigma T^{4}}{c}\right) \cdot \boldsymbol{u} \rightarrow-\frac{\rho_{0} u_{0}^{3}}{\ell_{0}} \nabla^{*} \cdot\left(\frac{4 \sigma T_{0}^{4}}{\rho_{0} u_{0}^{2} c} T^{* 4}\right) \cdot \boldsymbol{u}^{*}, \quad(\mathrm{~B} 1 \mathrm{~d}) \\
\nabla \cdot\left[\frac{\chi_{t h} \rho k_{B} \gamma}{m(\gamma-1)} \nabla T\right] \rightarrow \frac{\rho_{0} u_{0}^{3}}{\ell_{0}} \nabla^{*} \cdot\left[\frac{\chi_{t h} k_{B} T_{0} \gamma}{\ell_{0} m u_{0}^{3}(\gamma-1)} \rho^{*} \nabla^{*} T^{*}\right]
\end{gathered}
$$

$$
\begin{gathered}
\nabla \cdot\left\{\rho v\left[\nabla \boldsymbol{u}+(\nabla \boldsymbol{u})^{T}-\frac{2}{3}(\nabla \cdot \boldsymbol{u}) \underline{\boldsymbol{I}}\right]+\zeta(\nabla \cdot \boldsymbol{u}) \underline{\boldsymbol{I}}\right\} \cdot \boldsymbol{u} \\
\rightarrow \frac{\rho_{0} u_{0}^{3}}{\ell_{0}} \nabla^{*} \cdot\left\{\frac{\mu}{\rho_{0} u_{0} \ell_{0}}\left[\nabla^{*} \boldsymbol{u}^{*}+\left(\nabla^{*} \boldsymbol{u}^{*}\right)^{T}-\frac{2}{3}\left(\nabla^{*} \cdot \boldsymbol{u}^{*}\right) \underline{\boldsymbol{I}}\right]\right. \\
\left.+\frac{\zeta}{\rho_{0} u_{0} \ell_{0}}\left(\nabla^{*} \cdot \boldsymbol{u}^{*}\right) \underline{\boldsymbol{I}}\right\} \cdot \boldsymbol{u}^{*} \\
\boldsymbol{J} \cdot \boldsymbol{E} \rightarrow \frac{\rho_{0} u_{0}^{3}}{\ell_{0}} \frac{J_{0} E_{0} \ell_{0}}{\rho_{0} u_{0}^{3}} \boldsymbol{J}^{*} \cdot \boldsymbol{E}^{*} \\
\frac{\hbar^{2} \rho}{2 m^{2}} \nabla\left(\frac{\nabla^{2} \sqrt{\rho}}{\sqrt{\rho}}\right) \cdot \boldsymbol{u} \rightarrow \frac{\rho_{0} u_{0}^{3}}{\ell_{0}}\left(\frac{\hbar}{u_{0} \ell_{0} \sqrt{2} m}\right)^{2} \\
\times \rho^{*} \nabla^{*}\left(\frac{\nabla^{* 2} \sqrt{\rho^{*}}}{\sqrt{\rho^{*}}}\right) \cdot \boldsymbol{u}^{*} \\
-\frac{4 \sigma T^{4}}{3 c} \nabla \cdot \boldsymbol{u} \rightarrow-\frac{\rho_{0} u_{0}^{3}}{\ell_{0}}\left(\frac{4 \sigma T_{0}^{4}}{3 c u_{0}^{2} \rho_{0}}\right) T^{* 4} \nabla^{*} \cdot \boldsymbol{u}^{*}
\end{gathered}
$$

The factor $\rho_{0} u_{0}^{3} / \ell_{0}$ has been isolated from each term. If we divide through by this, we then obtain Equation (15).

\section{APPENDIX C}

\section{FULL EQUATIONS}

Here we give a full summary of all of the dimensionless equations of magneto-quantum-resistive hydrodynamics:

\section{C.1. Continuity Equation}

$$
\frac{\partial \rho^{*}}{\partial t^{*}}+\nabla^{*} \cdot \rho^{*} \boldsymbol{u}^{*}=0
$$

\section{C.2. Induction Equation}

$$
\begin{aligned}
\frac{\partial \boldsymbol{B}^{*}}{\partial t^{*}}= & \nabla^{*} \times\left(\boldsymbol{u}^{*} \times \boldsymbol{B}^{*}\right)+\frac{1}{R e_{M}} \nabla^{* 2} \boldsymbol{B}^{*}+\frac{1}{B i} \frac{\nabla^{*} p^{*} \times \nabla^{*} \rho^{*}}{\rho^{* 2}} \\
& +\frac{1}{N e} \nabla^{*} \times\left(\boldsymbol{B}^{*} \times \nabla^{*} T^{*}\right)
\end{aligned}
$$

\section{C.3. Momentum Equation (Optically Thick)}

$$
\begin{aligned}
& \rho^{*}\left(\frac{\partial \boldsymbol{u}^{*}}{\partial t^{*}}+\boldsymbol{u}^{*} \cdot \nabla^{*} \boldsymbol{u}^{*}\right)=-\nabla^{*}\left[p^{*}+\frac{1}{R} T^{* 4}\right] \\
& \quad+\frac{1}{\mathcal{H}_{Q}} \rho^{*} \nabla^{*}\left(\frac{\nabla^{* 2} \sqrt{\rho^{*}}}{\sqrt{\rho^{*}}}\right)+\nabla^{*} \cdot\left\{\frac { 1 } { R e } \left[\nabla^{*} \boldsymbol{u}^{*}+\left(\nabla^{*} \boldsymbol{u}^{*}\right)^{T}\right.\right. \\
& \left.\left.\quad-\frac{2}{3}\left(\nabla^{*} \cdot \boldsymbol{u}^{*}\right) \underline{\boldsymbol{I}}\right]+\frac{1}{\operatorname{Re}_{\zeta}}\left(\nabla^{*} \cdot \boldsymbol{u}^{*}\right) \underline{\boldsymbol{I}}\right\}+\frac{1}{\Omega_{R}} \rho_{C}^{*} \boldsymbol{E}^{*} \\
& \quad+\frac{1}{\Omega_{H}} \boldsymbol{J}^{*} \times \boldsymbol{B}^{*}
\end{aligned}
$$




\section{C.4. Momentum Equation (Optically Thin)}

$$
\begin{aligned}
\rho^{*} & \left(\frac{\partial \boldsymbol{u}^{*}}{\partial t^{*}}+\boldsymbol{u}^{*} \cdot \nabla^{*} \boldsymbol{u}^{*}\right)=-\nabla^{*} p^{*}+\frac{1}{\mathcal{H}_{Q}} \rho^{*} \nabla^{*}\left(\frac{\nabla^{* 2} \sqrt{\rho^{*}}}{\sqrt{\rho^{*}}}\right) \\
& +\nabla^{*} \cdot\left\{\frac{1}{\operatorname{Re}}\left[\nabla^{*} \boldsymbol{u}^{*}+\left(\nabla^{*} \boldsymbol{u}^{*}\right)^{T}-\frac{2}{3}\left(\nabla^{*} \cdot \boldsymbol{u}^{*}\right) \underline{\boldsymbol{I}}\right]\right. \\
& \left.+\frac{1}{\operatorname{Re}_{\zeta}}\left(\nabla^{*} \cdot \boldsymbol{u}^{*}\right) \underline{\boldsymbol{I}}\right\}+\frac{1}{\Omega_{R}} \rho_{C}^{*} \boldsymbol{E}^{*}+\frac{1}{\Omega_{H}} \boldsymbol{J}^{*} \times \boldsymbol{B}^{*}
\end{aligned}
$$

\section{C.5. Energy Equation (Optically Thick)}

$$
\begin{aligned}
\frac{\partial}{\partial t^{*}}\left(\rho^{*} \epsilon^{*}+\frac{\rho^{*} u^{* 2}}{2}+\frac{3}{R} T^{* 4}\right)+\nabla^{*} \cdot\left[\rho^{*} \boldsymbol{u}^{*}\left(\epsilon^{*}+\frac{u^{2}}{2}\right)+p^{*} \boldsymbol{u}^{*}\right] \\
=\nabla^{*} \cdot\left\{\frac{1}{\Pi_{\mathrm{thick}}} \frac{T^{* 3}}{\rho^{*}} \nabla^{*} T^{*}-\frac{3}{R} T^{* 4} \cdot \boldsymbol{u}^{*}+\frac{1}{P e} \frac{\gamma}{\gamma-1} \rho^{*} \nabla^{*} T^{*}\right. \\
+\frac{1}{\operatorname{Re}}\left[\nabla^{*} \boldsymbol{u}^{*}+\left(\nabla^{*} \boldsymbol{u}^{*}\right)^{T}-\frac{2}{3}\left(\nabla^{*} \cdot \boldsymbol{u}^{*}\right) \underline{\boldsymbol{I}}\right] \cdot \boldsymbol{u}^{*} \\
\left.\quad+\frac{1}{\operatorname{Re}_{\zeta}}\left(\nabla^{*} \cdot \boldsymbol{u}^{*}\right) \underline{\boldsymbol{I}} \cdot \boldsymbol{u}^{*}\right\}-\frac{1}{\Omega_{R}} \boldsymbol{J}^{*} \cdot \boldsymbol{E}^{*} \\
\quad+\frac{1}{\mathcal{H}_{Q}} \rho^{*} \nabla^{*}\left(\frac{\nabla^{* 2} \sqrt{\rho^{*}}}{\sqrt{\rho^{*}}}\right) \cdot \boldsymbol{u}^{*}-\frac{1}{R} T^{* 4} \nabla^{*} \cdot \boldsymbol{u}^{*}
\end{aligned}
$$

\section{C.6. Energy Equation (Optically Thin)}

$$
\begin{aligned}
& \frac{\partial}{\partial t^{*}}\left(\rho^{*} \epsilon^{*}+\frac{\rho^{*} u^{* 2}}{2}\right)+\nabla^{*} \cdot\left[\rho^{*} \boldsymbol{u}^{*}\left(\epsilon^{*}+\frac{u^{2}}{2}\right)+p^{*} \boldsymbol{u}^{*}\right] \\
& =\nabla^{*} \cdot\left\{\frac{1}{P e} \frac{\gamma}{\gamma-1} \rho^{*} \nabla^{*} T^{*}+\frac{1}{\operatorname{Re}}\left[\nabla^{*} \boldsymbol{u}^{*}+\left(\nabla^{*} \boldsymbol{u}^{*}\right)^{T}\right.\right. \\
& \left.\left.-\frac{2}{3}\left(\nabla^{*} \cdot \boldsymbol{u}^{*}\right) \underline{\boldsymbol{I}}\right] \cdot \boldsymbol{u}^{*}+\frac{1}{R e_{\zeta}}\left(\nabla^{*} \cdot \boldsymbol{u}^{*}\right) \underline{\boldsymbol{I}} \cdot \boldsymbol{u}^{*}\right\} \\
& -\frac{1}{\Omega_{R}} \boldsymbol{J}^{*} \cdot \boldsymbol{E}^{*}+\frac{1}{\mathcal{H}_{Q}} \rho^{*} \nabla^{*}\left(\frac{\nabla^{* 2} \sqrt{\rho^{*}}}{\sqrt{\rho^{*}}}\right) \cdot \boldsymbol{u}^{*}-\frac{1}{\Pi_{\mathrm{thin}}} \rho^{*} T^{* 4}
\end{aligned}
$$

\section{REFERENCES}

Alfvén, H. 1942, Natur, 150, 405 Biermann, L. 1950, ZNatA, 5, 65 Bildsten, L., \& Hall, D. M. 2001, ApJ, 549, 219

Bohm, D. 1952, PhRv, 85, 166

Braginskii, S. I. 1965, RvPP, 1, 205

Castor, J. I. 2004, Radiation Hydrodynamics (Cambridge: Cambridge Univ. Press)
Chapman, A., \& Cowling, T. G. 1970, The Mathematical Theory of NonUniform Gases (Cambridge: Cambridge Univ. Press)

Chevalier, R. A., Blondin, J. M., \& Emmering, R. T. 1992, ApJ, 392, 118

Connor, J. W., \& Taylor, J. B. 1977, NucFu, 17, 1047

Drake, R. P. 2006, High-Energy-Density Physics: Fundamentals, Inertial Fusion, and Experimental Astrophysics (New York: Springer)

Falize, É, Loupias, B., Ravasio, A., et al. 2011a, Ap\&SS, 336, 81

Falize, É, Michaut, C., \& Bouquet, S. 2011b, ApJ, 730, 96

Foster, J. M., Wilde, B. H., Rosen, P. A., et al. 2002, PhPl, 9, 2251

García Saiz, E., Gregori, G., Gericke, D. O., et al. 2008, NatPh, 4, 940

Gregori, G., Ravasio, A., Murphy, C. D., et al. 2012, Natur, 481, 480

Haas, F. 2011, Quantum Plasmas: An Hydrodynamic Approach (New York: Springer)

Haines, M. G. 1986a, PPCF, 28, 1705

Haines, M. G. 1986b, CaJPh, 64, 912

Hansen, J. F., Edwards, M. J., Froula, D., et al. 2005, Ap\&SS, 298, 61

Hartigan, P., Raymond, J., \& Hartmann, L. 1987, ApJ, 316, 323

Huba, J. D. 2002, Revised NRL Plasma Formulary (Washington, DC: Office of Naval Research)

Jeanloz, R., Celliers, P. M., Collins, G. W., et al. 2007, PNAS, 104, 9172

Katz, B., Budnik, R., \& Waxman, E. 2010, ApJ, 716, 781

Klein, R. I., Budil, K. S., Perry, T. S., \& Bach, D. R. 2003, ApJ, 583, 245

Kolmogorov, A. N. 1991, RSPSA, 434, 9

Krauland, C. M., Drake, R. P., Kuranz, C. C., et al. 2013a, ApJ, 762, L2

Krauland, C. M., Drake, R. P., Kuranz, C. C., et al. 2013b, PhPl, 20, 056502

Kritcher, A. L., Neumayer, P., Castor, J., et al. 2008, Sci, 322, 69

Kulsrud, R. M., \& Zweibel, E. G. 2008, RPPh, 71, 046901

Lai, D. 2001, RvMP, 73, 629

Landau, L. D., \& Lifshitz, E. M. 1959, Fluid Mechanics (Oxford: Pergamon Press)

Lifshitz, E. M., \& Pitaevskii, L. P. 1981, Physical Kinetics (Oxford Elsevier)

Manuel, M. J.-E., Li, C. K., Séguin, F. H, et al. 2013, PhPl, 20, 056301

McClarren, R. G., Drake, R. P., Morel, J. E., \& Holloway, J. P. 2010, PhPl, 17,093301

Meinecke, J., Doyle, H. W., Miniati, F., et al. 2014, NatPh, 10, 520

Miniati, F., et al. 2000, ApJ, 542, 608

Mostacci, D., Molinari, V., \& Pizzio, F. 2008, TTSP, 37, 589

Nishiguchi, A., Yabe, T., \& Haines, M. G. 1985, PhFl, 28, 3683

Pak, A., Divol, L., Gregori, G., et al. 2013, PhPl, 20, 056315

Reipurth, B., \& Bally, J. 2001, ARA\&A, 39, 403

Remington, B. A., Arnett, D., Drake, R. P., \& Takabe, H. 1999, Sci, 284,1488

Remington, B. A., Drake, R. P., \& Ryutov, D. D. 2006, RvMP, 78, 755

Robey, H., Perry, T. S., Klein, R. I., et al. 2002, PhRvL, 89, 085001

Ryutov, D. D., Drake, R. P., \& Kane, J. 1999, ApJ, 518, 821

Ryutov, D. D., Drake, R. P., \& Remington, B. A. 2000, ApJS, 127, 465

Ryutov, D. D., Kugland, N. L., Park, H. S., et al. 2012, PPCF, 54, 105021

Ryutov, D. D., Remington, B. A., Robey, H. F., \& Drake, R. P. 2001, PhPl, 8,1804

Savin, D. W., Brickhouse, N. S., Cowan, J. J., et al. 2012, RPPh, 75, 036901

Schmidt, R., Crowley, B. J. B., Mithen, J., \& Gregori, G. 2012, PhRvE, 85,046408

Shu, F. H. 1992, The Physics of Astrophysics Volume II: Gas Dynamics (Sausalito, CA: Univ. Science Books)

Sutherland, R. S., \& Dopita, M. A. 1993, ApJ, 88, 253

Tikhonchuk, V. T., Nicolaï, Ph., Ribeyre, X., et al. 2008, PPCF, 50, 124056

Tsakiris, G. D., \& Eidmann, K. 1987, JQSRT, 8, 353

Zel'dovich, Y. B., \& Raizer, Y. P. 1966, Physics of Shock Waves and Hightemperature Hydrodynamic Phenomena (New York: Academic Press)

Zingale, M., Almgren, A. S., Bell, J. B., Nonaka, A., \& Woosley, S. E. 2009, ApJ, 704, 196 